\title{
A Framework to Prioritise Health Research Proposals for Funding: Integrating Value for Money
}

\section{Haitham W. Tuffaha, Joanne Aitken, Suzanne Chambers \& Paul A. Scuffham}

\section{Applied Health Economics and Health} Policy

ISSN 1175-5652

Appl Health Econ Health Policy DOI 10.1007/s40258-019-00495-2
2013, Vol. 11, No. 4 (pp ONLINE FIRST

Applied Health Ecorrurircs and Health Policy

Current Opinion

New Anti-Rebate Legislation in South Korea

Review Article

Quantifying Benefit-K $K$ Syatic Review

Systematic Review
Assessing Cost Effectiverss

Original Research Articles

Original Research Articles os and health pol

Practical Approach for Calculating Reliable Cosss from Observational Data
Trade-off Between Costs and Quality of Care in Somatoform Rain Disorder

Factors Explaining Attitude Toward Social Health Insurance
Utilization of Venlafaxine after Introduction of Generís in Sweden

Utilization of Venlafaxine after Introduction of Generics in Sweden
Compliance and Healthcarc Costs in Parkinsonis Dissase
Pre-Diagnosis Costs in US Medicaid Patients with Altheimer's Disease

Pre-Diagnosis Cosss in US Medicaid Patients widh

Costs of Managing intracranial Aneurysms in traly

$\triangle$ Adis 
Your article is protected by copyright and all rights are held exclusively by Springer Nature Switzerland AG. This e-offprint is for personal use only and shall not be selfarchived in electronic repositories. If you wish to self-archive your article, please use the accepted manuscript version for posting on your own website. You may further deposit the accepted manuscript version in any repository, provided it is only made publicly available 12 months after official publication or later and provided acknowledgement is given to the original source of publication and a link is inserted to the published article on Springer's website. The link must be accompanied by the following text: "The final publication is available at link.springer.com". 


\title{
A Framework to Prioritise Health Research Proposals for Funding: Integrating Value for Money
}

\author{
Haitham W. Tuffaha ${ }^{1,2}$. $\cdot$ Joanne Aitken ${ }^{1,3} \cdot$ Suzanne Chambers ${ }^{1,3,4} \cdot$ Paul A. Scuffham $^{1,2}$
}

○) Springer Nature Switzerland AG 2019

\begin{abstract}
When making funding decisions, research organisations largely consider the merits (e.g. scientific rigour and feasibility) of submitted research proposals; yet, there is often little or no reference to their value for money. This may be attributed to the challenges of assessing and integrating value of research into existing research prioritisation processes. We propose a framework that considers both the merits of research and its value for money to guide health research funding decisions. A practical framework is developed based on current processes followed by funding organizations for assessing investigatorinitiated research proposals, and analytical methods for evaluating the expected value of research. We apply the analytical methods to estimate the expected return on investment of two real-world grant applications. The framework comprises four sequential steps: (1) initial screening of applications for eligibility and completeness; (2) merit assessment of eligible proposals; (3) estimating the expected value of research for the shortlisted proposals that pass the first two steps and ranking of proposals based on return on investment; and (4) selecting research proposals for funding. We demonstrate how the expected value for money can be efficiently estimated using certain information provided in funding applications. The proposed framework integrates value-for-money assessment into the existing research prioritisation processes. Considering value for money to inform research funding decisions is vital to achieve efficient utilisation of research budgets and maximise returns on research investments.
\end{abstract}

\section{Background}

Health research is essential to improve individual and population health by guiding decision making at the clinical and policy levels. Therefore, governments and research organisations allocate considerable resources to support clinical trials and other research studies. For instance, the Australian Government established the Medical Research Future Fund (MRFF) with a total budget of $\$ 20$ billion (all values

Electronic supplementary material The online version of this article (https://doi.org/10.1007/s40258-019-00495-2) contains supplementary material, which is available to authorized users.

Haitham W. Tuffaha

haitham.tuffaha@griffith.edu.au

1 Menzies Health Institute Queensland, Griffith University, Gold Coast, QLD, Australia

2 School of Medicine, Centre for Applied Health Economics, Griffith University, Nathan 4111, QLD, Australia

3 Cancer Council Queensland, Spring Hill, QLD, Australia

4 Faculty of Health, University of Technology Sydney, Sydney, NSW, Australia are given in Australian dollars) to drive innovation, improve delivery of healthcare, and enhance the efficiency and effectiveness of the health system in Australia [1]. However, research budgets are usually insufficient to fund all worthy applications, and funding organisations must therefore make decisions about the best way to prioritise and select competing research proposals. These decisions are usually made based on the assessments of the merits (e.g. scientific rigour and feasibility) of the submitted research proposals, as judged by expert panels [2,3], with little or no reference to explicit estimates of the value for money of proposed research programs [3]. Nevertheless, consideration of the expected costs and benefits of research proposals is fundamental to achieve efficient utilisation of research budgets and maximise returns on research investments.

Analytic approaches have been proposed to quantify the value of evaluative research (i.e., clinical trials and cohort studies) [3,4]. The underlying principle of these approaches is to estimate the expected benefits of research for improving health outcomes (e.g. survival or quality of life), which can be expressed in monetary terms using a willingness-to-pay value for an additional unit of health outcome (e.g. $\$ 50,000$ per life-year gained). This expected benefit is compared to 


\section{Key Points for Decision Makers}

Research organisations must make decisions about the best way to prioritise and select competing research proposals for funding. These decisions are often informed by the qualitative merits of research proposals.

Consideration of the expected costs and benefits of research proposals (i.e., value for money) is fundamental to achieve efficient utilisation of research budgets and maximise returns on research investments.

We propose a framework that considers both the merits of research and its value for money to guide health research funding decisions.

the expected costs of conducting the proposed research to inform if this research proposal is cost-effective. Unfortunately, the application of quantitative approaches to prioritise research funding decisions in practice is limited [5, 6]. This may be attributed to two main reasons. First, the burden associated with quantitative approaches in terms of the availability of the technical skills within funding organisations and the additional time required to conduct the analyses [6, 7]; notably, the peer-review process for the National Health and Medical Research Council (NHMRC) Project Grant scheme in 2017 took approximately 6 months to evaluate 3500 applications [2]. Second, it is unclear how to incorporate the value-for-money criterion into the existing prioritisation process alongside other attributes (i.e., criteria) such as research scientific rigor and team quality and capability.

The aim of this paper was to propose a practical framework that considers both the merits of research and value for money to guide health research funding decisions by organisations in Australia. We describe, in some detail, how to quantify the value for money for assessing grant applications, and then provide two worked examples.

\section{The Framework}

In order to incorporate value for money into existing processes for research prioritisation in Australia, the framework is informed by: (1) current processes by Australian funding organisations for assessing investigator-initiated research proposals, (2) international approaches and good practices for research prioritisation, and (3) common principles for evaluating value for money of research studies. The current practices by Australian funding organisations are based on the peer-review principles for NHMRC grants and other major funding schemes listed on the Australian Competitive Grants Register [3, 8-10]. Typically, submitted proposals are received and screened for eligibility, assessed and scored against predefined criteria, ranked, and finally selected based on discussions among the review panel members [3, 8-10]. This information is complemented by best international practices for research prioritisation methods and criteria reported in the literature [11-13]. The value-for-money component was based on the value of information-analysis approach [6, 7, 14-17], and value of improved implementation [18-22], whereby the expected benefits of a research study are explicitly compared with its expected costs.

A framework for prioritising research proposals for funding is presented in Fig. 1. The framework comprises four steps: (1) initial screening of applications; (2) assessment of research proposals' merits; (3) estimating the value for money of research proposals; and (4) selecting research proposals for funding.

\subsection{Initial Screening}

The first step after receiving applications for funding involves screening proposals for eligibility and completeness. This step is done by administrators to verify that submitted proposals meet the eligibility criteria of the funding scheme and that all necessary information is provided in the submitted applications. Certain criteria can be applied at this stage such as relevance to the grant scheme, completeness of information and conformity to application guidelines. At this stage, ineligible or incomplete proposals are eliminated.

\subsection{Assessment of Proposals' Merits}

The merits of the submitted research proposals are assessed using a predefined set of criteria (i.e., attributes). The selected criteria should represent the merits that stakeholders believe should be present in the proposed research study. Relevant criteria can be identified in a number of ways including literature reviews, focus groups or surveys. In general, selection criteria can be grouped into representative domains such as relevance (i.e., why should we do it?, including the burden of disease, equity considerations and knowledge gap), appropriateness (i.e., should we do it?, including scientific rigour and suitability of methods), significance of research outcomes (i.e., what will we get out of it?, including impact, capacity building and innovation), feasibility (i.e., can we do it?, including team quality and research environment) $[11,13]$. Importantly, the chosen criteria should be clear and well defined as well as being independent of each other and non-redundant to avoid double counting $[11,13,23]$. The way the proposals are assessed against these criteria may vary according to the organization's processes and available resources. Applications can be shortlisted by excluding proposals that fail to meet any of the pre-specified criteria. Alternatively, submitted proposals can 
Fig. 1 Framework for prioritising research proposals for funding

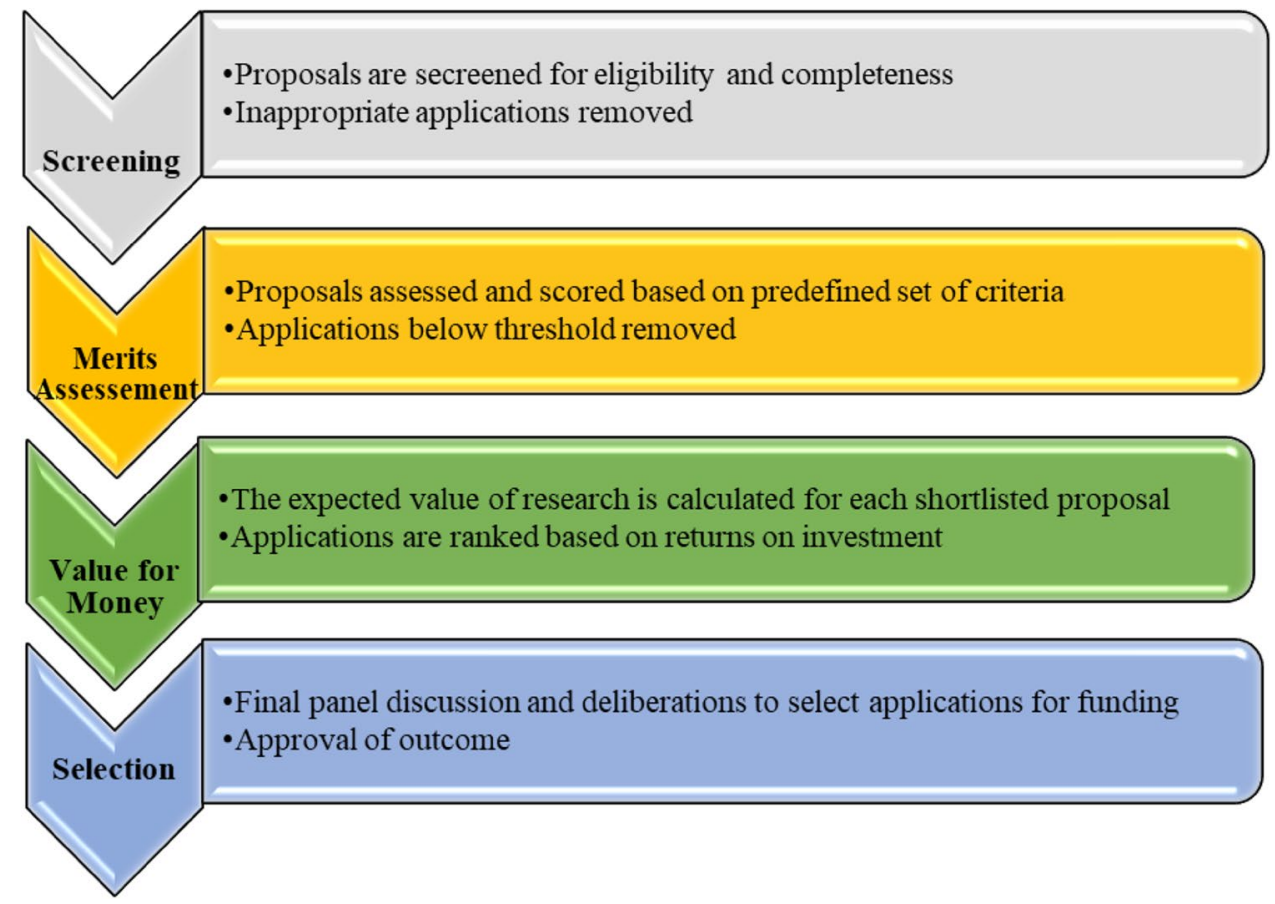

be assessed and scored against these criteria with each criterion, or group of criteria, being assigned a weight reflecting its relative importance. This can be done using a structured and explicit approach such as multiple criteria decision analysis (MCDA) [23-25]. At the end of this step, a short list of proposals that have the required merits will move to the next step. To avoid double counting in research-value assessment, the scores from this step are not carried forward to the next step where the shortlisted proposals will be evaluated for their value for money.

\subsection{Value for Money}

In this stage, the expected value (i.e., benefits) of each shortlisted proposal is estimated. In estimating the additional value of a new research study, the expected benefits of two states are compared: (1) a factual state in which research takes place, and (2) a counterfactual state, in which research is not conducted [4].

Research informs decision making in two ways. First, collecting more information may reduce the chance of making misguided decisions when the existing evidence is uncertain $[12,13]$. Misguided decisions may lead to healthcare costs such as wasting resources on ineffective or unsafe interventions. Reducing uncertainty by collecting additional evidence better informs decision making, and in doing so avoids the costly consequences of suboptimal decisions $[15,17]$. Second, research may influence the implementation of interventions (e.g. changing practice) by adding to information and evidence. Collecting further evidence has the potential to improve health outcomes by encouraging the implementation of the most effective and/or cost-effective intervention [18-20, 22, 26]. Thus, the expected value of a research study is the sum of its expected value in reducing decision uncertainty and its expected value in improving implementation [18].

These expected research benefits are scaled up by considering the population expected to benefit from research findings and the evidence durability (i.e., the time over which evidence is useful). Then, the expected value of research at the population level is compared to the expected research costs to estimate the return on investment of each proposal.

\subsubsection{Value of Research in Reducing Decision Uncertainty}

Under budget constraints, the decision to adopt a new intervention (i.e., to translate or implement the intervention) is typically guided by its ability to provide value for money (i.e., cost-effectiveness). Specifically, the costs and benefits of the new intervention are compared with the costs and benefits of alternative options. The additional benefit of the new intervention can be converted to dollar value by multiplying the expected improvement in health by a monetary value per unit of health gained (e.g. $\$ 50,000$ per life-year gained). Nevertheless, any evidence is never certain because we do not know the true values of effectiveness and cost estimates, and thus any decision based on this evidence is also uncertain [27]. Under this uncertainty, making the wrong decision 
could lead to costly consequences to the healthcare system (i.e., the opportunity cost of adopting a suboptimal option). Thus, information is valuable because it reduces the risk of making wrong decisions.

Value of Information (VOI) analysis is a method to estimate the expected costs of decision uncertainty, and, therefore, the value of research aimed at reducing this uncertainty. In estimating the cost of decision uncertainty, VOI considers two factors: (1) the probability of making the wrong decision (e.g. the probability the intervention is not cost-effective), and (2) the consequences of this error (e.g. opportunity loss of adopting a suboptimal intervention) [7, 15, 28]. If we had perfect information, we would avoid the cost of uncertainty and maximise the expected monetary benefit of our decision. The difference between the expected benefit of a decision based on perfect information (i.e., no uncertainty) and the benefit made based on available evidence (i.e., with uncertainty) is the Expected Value of Perfect Information (EVPI) [29]. The EVPI is the value of the additional information that would resolve all uncertainty; however, it is almost impossible in practice to have perfect information; and hence, what the EVPI represents is the maximum value (i.e., the upper-bound) of research benefit. The Expected Value of Sample Information (EVSI), on the other hand, estimates the value of a clinical trial of a given sample size in reducing, but not resolving, decision uncertainty, which can be calculated as the difference between the expected benefit from a future clinical trial and the benefit based on current evidence [15]. Clearly, the data collected from additional research is not known at this stage but could be predicted by simulation [30, 31].

Typically, VOI requires the development of a decisionanalytic model to assess the cost-effectiveness and characterise decision uncertainty; however, research funding organisations may not have the time and/or capacity to build models to evaluate all research proposals. A rapid approach is to use the minimal modelling for VOI calculation as suggested by Meltzer et al. [32]. This approach can be performed when the outcomes (and costs) of interest and the uncertainty around these estimates are sufficiently reported in prior evidence to inform a decision about the benefits of alternative interventions; for example, when a prior study captures comprehensive outcomes such as improvement in survival or quality-adjusted life-years (QALYs) gained. Even when intermediate outcomes are reported (e.g. progressionfree survival), these can be extrapolated using external data to obtain comprehensive measure of outcome [32,33].

To calculate the expected value of reducing uncertainty, grant applications should provide the following information based on current evidence: the primary outcome(s) of the intended research, description of existing evidence including type (e.g. pilot study, meta-analysis, or expert opinion) and sample size, the effectiveness of the comparator (e.g. baseline risk), relative effectiveness of the intervention (e.g. relative risk, risk difference), the estimated costs of the intervention and comparator, uncertainty around effectiveness and cost estimates (e.g. standard error or confidence interval), willingness-to-pay for an additional unit of effectiveness (e.g. per QALY gained or event avoided), and the sample size and follow-up duration of the new clinical trial. Table 1 summarises the information required in funding applications.

\subsubsection{Value of Research in Improving Implementation}

Based on existing evidence, there is a current level of uptake (i.e., implementation) of the interventions being researched; this can range from 0 (no implementation) to 1 (perfect implementation). Perfect implementation cannot be achieved in practice; however, the results of new research can optimise the uptake of these interventions, leading to the implementation of the cost-effective intervention and disinvestment in interventions that do not provide value for money. Of course, there are other ways to optimise implementation other than research (e.g. knowledge transfer and incentives), but what we are interested in is the expected change in implementation that can be attributed to the results of new research $[18,20]$. Thus, the value of research in changing the level of implementation is the difference between the expected benefits of the intervention from an improved level of implementation and the benefits based on current uptake levels $[18,20]$. For this calculation, the applications should provide the current level of uptake of the researched interventions and the expected level of uptake when the new research results are out. The expected level of uptake in the future can be obtained from elicitation of expert belief conditional on the strength of evidence, realistically varying implementation levels over time to allow for evidence diffusion $[18,20]$.

\subsubsection{Expected Value of Research at the Population Level}

The expected value of research is the sum of the expected value of reducing uncertainty and the expected value of improving implementation [18]. This value is per patient; however, to calculate total research value at the population level, the expected value of research is multiplied by the population expected to benefit from the intervention over a given number of years. This duration should reflect the time over which the results from this research remain useful. Because these benefits happen in the future, expected benefits are discounted at an appropriate discount rate (e.g. annual rate of 5\%). The application should provide data on the incidence and/or prevalence population, depending on the nature of the condition being addressed (i.e., chronic or acute) as well as the number of years over which 
Table 1 Summary of the information required to calculate expected value of research

\begin{tabular}{|c|c|c|}
\hline Domains & Inputs & Clarifying statements \\
\hline \multirow[t]{7}{*}{ Value of resolving uncertainty } & Primary outcome(s) of the new research & $\begin{array}{l}\text { The primary outcome in the research proposal (e.g. survival, } \\
\text { quality of life, event avoided) }\end{array}$ \\
\hline & Existing evidence & $\begin{array}{l}\text { The source of current evidence (e.g. meta-analysis, systematic } \\
\text { review, clinical studies or expert opinion) }\end{array}$ \\
\hline & Effectiveness of the comparator/control & $\begin{array}{l}\text { The effectiveness of the comparator (e.g. baseline probability or } \\
\text { mean estimate of the outcome of evaluated), with standard error } \\
\text { or confidence interval }\end{array}$ \\
\hline & Relative effectiveness of the intervention & $\begin{array}{l}\text { The relative effectiveness of the intervention (e.g. relative risk or } \\
\text { risk difference), with standard error or confidence interval }\end{array}$ \\
\hline & Cost of the comparator & The expected cost of the comparator \\
\hline & Cost of the intervention & The expected cost of the intervention or incremental cost \\
\hline & Willingness-to-pay & Indicate the monetary value of one unit of effectiveness \\
\hline \multirow[t]{2}{*}{$\begin{array}{l}\text { Value of improved } \\
\text { implementation }\end{array}$} & Current level of implementation/uptake & $\begin{array}{l}\text { The current level of implementation/uptake based on existing } \\
\text { evidence }\end{array}$ \\
\hline & Expected level of implementation/uptake & $\begin{array}{l}\text { The expected level of implementation/uptake with the new } \\
\text { research }\end{array}$ \\
\hline \multirow{3}{*}{$\begin{array}{l}\text { Population benefiting from } \\
\text { research findings }\end{array}$} & Annual incidence & Incidence of the condition being researched \\
\hline & Prevalence & Prevalence of the condition being researched \\
\hline & Durability of information & $\begin{array}{l}\text { Time in years over which the findings from the new research is } \\
\text { useful }\end{array}$ \\
\hline \multirow[t]{3}{*}{ Other inputs } & Time to report research results & Time in years for research results to report \\
\hline & Discount rate & The rate of discounting future benefits and costs (e.g. 5\%) \\
\hline & Direct research cost & Research study budget including fixed and variable cost \\
\hline
\end{tabular}

the intervention is expected to remain useful in practice. Although a time horizon between 5 and 10 years is commonly used in the literature, better estimation of the time for the intervention to remain useful can be based on empirical evidence (e.g. historical data) or elicited from experts [14].

\subsubsection{Research Cost-Benefit Comparison}

Total research cost has two components, one financial (i.e., direct costs) and the other reflecting opportunity loss [20]. Direct costs comprise the fixed costs of setting up a trial, and variable costs, which is the cost per patient. Direct costs of research are obtained from the estimated budget in the grant application. The opportunity cost component is incurred by patients allocated to the control arm who will not benefit from the new intervention [26, 34]. The expected value of research at the population level is compared to the expected total research cost, the difference is the expected monetary gain of research. If the net gain is positive, then this proposed research study is cost-effective [20, 35, 36]. The return on investment is the expected net gain divided by the research cost. Proposals that are not cost-effective may be excluded and the rest of the proposals may be ranked according to their returns on investment. Grant applications should provide the expected research budget and the duration of the trial until the results are available.

\subsection{Selecting Proposals for Funding}

The final step in the framework involves the discussion and deliberation among panel members to reach consensus on which applications to fund. At this stage of the research prioritisation process, a short list of high-quality and costeffective proposals is considered. In making the final funding decisions, panel members will take into consideration both the merits and return-on-investment of competing proposals together with any other considerations (e.g. total budget available or equity). For instance, the panel may decide to fund applications with the highest return-on-investment and until the allocated budget is consumed. The final decision will be approved by the final decision maker (e.g. Minister of Health).

\section{Examples of Value-of-Research Calculations}

Two examples are presented to illustrate expected value-ofresearch calculation for two funding proposals: (1) a randomised controlled trial of a nurse-led multimodal intervention for meeting the psychosocial care needs of men with advanced prostate cancer, and (2) a randomised controlled trial of negative pressure wound therapy (NPWT) in 
preventing surgical site infection in obese women undergoing caesarean section. The calculations were performed in Microsoft Excel ${ }^{\circledR}$ (Version 2013). Technical details of the calculations are provided in Appendix 1.

\subsection{Nurse-led Multimodal Intervention in Men with Advanced Prostate Cancer}

Men with advanced prostate cancer have higher levels of psychological distress and poorer quality of life [37, 38]. Currently, there are no proven effective ways to improve psychological adjustment and quality-of-life outcomes for men with this condition. A randomised controlled trial is proposed to evaluate the effectiveness and cost-effectiveness of a tele-based nurse-led multimodal psychosocial intervention compared with usual care. The proposed study will have two arms: (1) minimally enhanced usual care (standard medical management with a package of patient education materials); and (2) nurse-led multimodal psychosocial care delivered over four weekly telephone sessions with a final booster follow-up session 1 month subsequently. The study will include 250 men (i.e., 125 in each arm) with advanced prostate cancer. The duration of the trial will be four years and the total requested budget is approximately $\$ 0.85$ million.

Available evidence comes from a subgroup $(n=110)$ in a trial evaluating the cost and effectiveness of an individualised cognitive behavioural intervention comprising five sessions of telephone-based counselling compared with a nurse-led single session of self-management in highly distressed cancer patients [39]. The intervention group had 0.037 QALYs gained (95\% CI - 0.045-0.118) with an incremental cost of $-\$ 322$ (95\% CI - 2609-1964). At $\$ 50,000$ per QALY gained the incremental net monetary benefit was \$2172 (95\% CI - 2610-8551) [40]. The population expected to benefit from the results of the study is based on around 32,000 patients, based on a prevalent population of 25,000 patients with advanced prostate cancer together with $8 \%$ of newly diagnosed prostate cancer cases $(17,000)$ progressing into advanced disease every year [41]. The research team expect an uptake rate from $10 \%$ in year one up to $50 \%$ in year five (average $30 \%$, assuming $10 \%$ increment every year), if the expected benefits were confirmed in the proposed larger trial.

Simulating the expected benefit from a clinical trial of 250 patients, the expected value of reducing uncertainty is $\$ 215$ per patient (Appendix 1). The benefit from improved implementation is $\$ 650$ (i.e., $(30-0 \%) \times \$ 2172$ ) per patient, and the expected value of research is around $\$ 865$ per patient (i.e., $\$ 215+\$ 650$ ). The expected value of research at the population level, over 5 years, is around $\$ 27.70$ million (i.e., $\$ 865 \times 32,000$ ). With a research budget of $\$ 0.85$ million and an opportunity cost for patients in the control arm of $\$ 0.27$ million (i.e., $125 \times \$ 2172$ ), the total research cost is
$\$ 1.12$ million. Thus, the expected net monetary gain of the proposed clinical trial is around $\$ 26.60$ million (i.e., $\$ 27.7$ million-\$1.12 million), which is a return on investment of approximately $2370 \%$.

\subsection{Negative Pressure Wound Therapy in Obese Women Undergoing Caesarean Section}

Obese women undergoing caesarean section are at increased risk of postoperative infection. NPWT may be useful to prevent closed surgical incision complications including surgical site infection (SSI); however, the evidence on its effectiveness in this population is limited. A randomised controlled trial has been proposed to assess the effect of NPWT on SSI in obese women undergoing elective caesarean section. The trial will include 400 patients (200 in each arm) who will be randomised to the NPWT or the standard hydrocolloid dressing. Trial duration is 4 years and the total budget requested is approximately $\$ 2$ million.

From available evidence, the baseline risk of SSI in the control group is $24 \%$ ( $\mathrm{SE}=24 \%$ ), which is based on a pooled estimate from observational studies reporting SSI in obese women undergoing caesarean surgery [42-45]. The relative risk (RR) of SSI in the intervention is obtained from the combined results of two small trials $(n=173)$ at 0.73 (95\% CI 0.39-1.32) [46, 47]. The cost of the NPWT device is $\$ 175$ and for the hydrocolloid dressing $\$ 7.50$. The willingness-to-pay threshold for an SSI event avoided is set at approximately $\$ 4000$, based on the weighted average cost of SSI treatment and reduced quality of life, which is assumed to reflect the maximum amount of money the decision maker is willing to pay to avoid an SSI event [48]. The intervention is expected to be implemented after the end of the trial and to be used in practice for at least 5 years. The population expected to benefit is estimated at 20,000 annually, since obese women undergoing caesarean surgery represent $20 \%$ of the 100,000 caesarean sections performed every year in Australia, with a population of 90,000 (discounted over 5 years) $[48,49]$. Expert advice indicates that the current level of implementation of NPWT is $25 \%$ and that a positive RCT may increase uptake from $30 \%$ in year 1 to $70 \%$ by year 5 (average $50 \%$, assuming $10 \%$ increment every year).

The net monetary benefit of NPWT compared with colloid dressing can be calculated as the difference between the SSI probabilities between the two arms times the willingness-to-pay threshold per an SSI event avoided, minus the difference in cost between the two interventions. Based on existing evidence, the incremental net benefit is $\$ 90$ (i.e., $24 \%-(0.73 \times 24 \%) \times \$ 4000-\$ 167.50)$. Simulating the expected benefit from a clinical trial of 400 patients, the expected value of reducing uncertainty is $\$ 51$ per patient. The expected value of improvement in implementation is $\$ 23$ (i.e., $(50 \%-25 \%) \times \$ 90)$ per patient. Thus, the expected 
value of research per patient is $\$ 74$ (i.e., $\$ 51+\$ 23$ ) and the expected value of research at the population level is around $\$ 6.6$ million (i.e., $\$ 74 \times 90,000)$. With a research budget of $\$ 2$ million and an opportunity cost of research of $\$ 18,000$ (i.e., $\$ 90 \times 200$ ), the total research cost is $\$ 2.02$ million. Thus, the expected net benefit of research is around $\$ 4.58$ million (i.e., $\$ 6.6$ million-\$2.02 million) and the expected return on investment is around $227 \%$. Table 2 summarises the inputs and results of the expected value of research calculations.

\section{Discussion}

Prioritising research proposals for funding is essential to ensure that limited research budgets are allocated to highquality and cost-effective projects. We propose a comprehensive framework that considers both the merits of research (e.g. team capability, study feasibility) and its value for money to guide research funding decisions. Importantly, the framework allows for comparison of research proposals across different research topics. The framework is aligned with most of the current evaluation processes whereby applications are received, screened for eligibility and completeness, and assessed against pre-defined criteria before a final decision is made through discussion and deliberation among panel members. The value-for-money component is incorporated in a later stage in the evaluation process and can be efficiently using information provided in funding applications.

In the first two steps in the framework, competing proposals are assessed against a pre-defined set of criteria such as scientific rigour, team quality, and research feasibility. This transparent approach provides clarity about the assessment criteria, and allows for the incorporation of relevant and important research attributes from the perspective of various stakeholders including patients, researchers and funders. Of note, considering the needs of stakeholders has been suggested as an important step to reduce research wastage [50]. Considering value for money as an attribute alongside other

Table 2 Expected value-of-research calculations for the nurse-led intervention in prostate cancer and NPWT in caesarean section

\begin{tabular}{|c|c|c|}
\hline & Nurse-led intervention in prostate cancer & NPWT in caesarean section \\
\hline \multicolumn{3}{|l|}{ Inputs } \\
\hline Primary outcome of the new clinical trial & QALY & RR of SSI \\
\hline Source and size of existing evidence & RCT (110 patients) & Pooled RCTs (173 patients) \\
\hline Effectiveness of the control & 0.577 QALYs & $24 \%$ baseline risk of SSI \\
\hline Relative effectiveness of the intervention & $0.037(95 \% \mathrm{CI}-0.045-0.118)$ & 0.73 (95\% CI 0.39-1.32) \\
\hline Willingness-to-pay & $\$ 50,000 / \mathrm{QALY}$ & $\$ 5000 /$ SSI avoided \\
\hline Cost of the comparator & $\$ 4095$ & $\$ 7.5$ (assumed fixed) \\
\hline Cost/incremental cost of the intervention & $\$-322(95 \%$ CI $-\$ 2609-\$ 1964)$ & $\$ 175$ (assumed fixed) \\
\hline Sample size of the new trial & 250 & 400 \\
\hline Current level of implementation/uptake & $0 \%$ & $25 \%$ \\
\hline Expected level of implementation/uptake & $30 \%$ & $50 \%$ \\
\hline Annual incidence & 1300 & 20,000 \\
\hline Prevalence & 25,000 & - \\
\hline Time to report research results & 4 years & 4 years \\
\hline Durability of information & 5 years & 5 years \\
\hline Research budget & $\$ 850,000$ & $\$ 2000,000$ \\
\hline Discount rate & $5 \%$ & $5 \%$ \\
\hline \multicolumn{3}{|l|}{ Outputs } \\
\hline Expected value of reduced uncertainty/patient & $\$ 215$ & $\$ 51$ \\
\hline Value of improved implementation/patient & $\$ 650$ & $\$ 23$ \\
\hline Expected research value/patient & $\$ 865$ & $\$ 74$ \\
\hline Expected research value/population & $\$ 27,680,000$ & $\$ 6600,000$ \\
\hline Expected total research cost & $\$ 1120,000$ & $\$ 2020,000$ \\
\hline Expected net benefit of research & $\$ 26,560,000$ & $\$ 4580,000$ \\
\hline Return on investment & $2370 \%$ & $227 \%$ \\
\hline
\end{tabular}

$N P W T$ negative pressure wound therapy, $Q A L Y$ quality-adjusted life-years, $S S I$ surgical site infection, $R R$ relative risk, $R C T$ randomised controlled trial, $C I$ confidence interval 
criteria (e.g. using MCDA) to calculate a composite score to rank proposals may not be ideal because it is burdensome to estimate value for money for all applications, and, importantly, it does not consider the opportunity cost forgone when applications that are not cost-effective are prioritised because they are deemed to be of high quality and/or feasibility. This can be overcome by assigning monetary values to all attributes, but this task is challenging and may not be acceptable by stakeholders. For instance, what monetary value should be assigned to equity or innovation? Adding the value-for-money aspect to a separate and sequential stage of the evaluation process ascertains that only a shortlist of high-quality and feasibility applications are evaluated for value for money.

To quantify the value of research, the framework combines both the value of reducing uncertainty (i.e., VOI) and value of research in improving implementation. VOI is a rigorous analytical approach that is gaining increasing attention to prioritise research studies and to link research to decision makers' needs (e.g. the need for additional research to guide reimbursement decisions); however, the value of reducing decision uncertainty cannot be separated from the impact of strengthening evidence on improving implementation of research findings [18, 20, 22]. Our approach builds on the observations of other researchers about the relationship between strength of evidence and implementation, and how ignoring the benefits of improved implementation could underestimate the expected value of research $[18,20$, 22]. This step will improve research value and reduce wastage by considering the expected benefit of the intervention compared with other alternatives, uncertainty in existing evidence, the potential of future implementation, and the burden of the disease when the expected value or research is scaled up to the population level. Moreover, this approach facilitates linking research funding to reimbursement decision needs (e.g. collecting evidence to inform funding decisions of novel drugs) [51].

The biggest challenge for incorporating value for money in research prioritisation frameworks has been the complexity and burden of analysis to estimate VOI $[5,6,52]$. In our framework, only shortlisted applications will be evaluated for value for money, which will reduce the burden of this vital step. Moreover, the framework adopts the minimal modelling approach to calculate value of information, which does not require sophisticated modelling [32, 33]. In the framework, applicants will be providing key inputs from existing evidence or expert opinion. These inputs can be verified at a step before value of research calculation (e.g. during the assessment of proposals in Steps 1 and 2). Value of research calculations can be done in a short time once the inputs are provided, which we have demonstrated in two real-world examples for actual research grant applications.
Moreover, and to facilitate calculations, a tool can be programmed in a suitable software whereby relevant inputs are entered and the estimates are calculated in seconds [53, 54]. The tool will provide a common interface between funders and researchers, which will improve transparency and acceptability of the outcomes.

This work is the first step in a larger project to develop a research evaluation tool to enable research funding organisations and researchers to prospectively assess value for money of research projects. Three research organisations were involved in the development of this framework, namely, Menzies Health Institute Queensland, Cancer Council Queensland and the Prostate Cancer Foundation of Australia. The face validity of the framework has been validated by leading researchers at these organisations. The next step is to develop a programmable tool to calculate value of research and to test the tool on a larger number of proposals from other funding organisations to evaluate its feasibility and impact in practice. It is worth mentioning that the analytical approaches to calculate value for money have been focused on applied and evaluative research (e.g. clinical trials and cohort studies); nevertheless, research funding covers a wide range of research programs including basic research, and, therefore, it would be useful to explore how value for money can be evaluated for a wider scope of programs. Although our framework takes an Australian healthsystem perspective, we believe that this approach is generalizable to other jurisdictions where researcher-initiated proposals are prioritised and selected for funding. Even if cost-effectiveness of researched interventions is not required in certain jurisdictions, value of research can be still calculated using health measures (e.g. life-years gained) rather than net monetary benefit [55].

The minimal approach we use to calculate VOI has some limitations. Namely, the availability of the data required to conduct the analyses, the need to use intermediate outcomes and the need to establish an appropriate willingness-to-pay thresholds. Furthermore, this approach does not capture all the costs and benefits (parameters) and is most suitable when there is a comparator arm. To overcome these limitations, certain assumptions have to be made such as the usefulness of existing evidence for the minimal modelling approach and the expected future implementation of the intervention. These assumptions might introduce biases to the results and undermine research prioritisation recommendations. To mitigate this risk, the quality of evidence should be carefully assessed to ensure that only good quality and relevant evidence is used in the calculations. Additionally, and in the absence of empirical evidence, expert opinion should be elicited using transparent and structured approaches [56, 57]. The robustness of the results to these assumptions can be tested in sensitivity analyses and presented to decision 
maker. It should be noted that when the minimal modelling approach is feasible (e.g. pilot study reporting comprehensive outcomes, or expert opinion to empirically characterize the relationship between the trial's primary endpoint and a comprehensive measure of health outcomes), it allows VOI analyses to be undertaken for the purpose of research prioritisation when the expertise to perform a full modelling approach is not available and/or when the results of a VOI analysis are required very quickly. Of note, VOI analyses based on full modelling may be considered by organisations interested in having more accurate estimates of research value for money (e.g. for research proposals asking for high budgets). This task can be outsourced to specialised research groups to undertake; however, expertise and time required to conduct VOI analyses based on full modelling should be carefully considered. Finally, there might be a concern that research studies targeting certain diseases and small but marginalised population may not be selected for funding based on their unfavourable cost-effectiveness figures. In this regard, it should be emphasised that cost-effectiveness should not be the only consideration for making research funding decisions. As indicated in the final step in our proposed framework, the decision will be made through discussion and deliberation among panel members where other important aspects (e.g. equity) are considered.

\section{Conclusion}

We have proposed a comprehensive framework that integrates value for money into the existing research prioritisation processes for funding organisations. Considering value for money alongside other important criteria to inform research funding decisions is essential achieve efficient utilisation of research budgets and maximise returns on research investments.

Acknowledgements The authors thank Prof. Wendy Chaboyer and Prof. Brigid Gillespie for the information they provided about the NPWT case.

Authors' contributions All authors have contributed to the conception of the article. HT drafted the manuscript and conducted the analysis. SC, JA and PS were involved in drafting the manuscript and revising it critically. All authors approved the final version of the manuscript.

\section{Compliance with Ethical Standards}

Conflict of interest Haitham Tuffaha, Joanne Aitken, Suzanne Chambers, and Paul Scuffham have no conflict of interest to declare.

Funding HT is supported by an Australian National Health and Medical Research Council (NHMRC) fellowship (GNT1121232). The authors acknowledge Menzies Health Institute Queensland, Cancer Council Queensland and the Prostate Cancer Foundation of Australia for funding this project.

\section{References}

1. DoH. Medical Research Future Fund. https://beta.health.gov.au/ initiatives-and-programs/medical-research-future-fund/about-themrff. Accessed 30 June 2018.

2. Peer Review Consultation Paper. https://consultations.nhmrc.gov. au/public_consultations/nhmrc-grant-program-review. Accessed 01 Dec 2017.

3. Tuffaha HW, Andronis L, Scuffham PA. Setting Medical Research Future Fund priorities: assessing the value of research. Med J Aust. 2017;206(2):63-5.

4. Andronis L. Analytic approaches for research priority-setting: issues, challenges and the way forward. Expert Rev Pharmacoecon Outcomes Res. 2015;15(5):745-54.

5. Tuffaha HW, Gordon LG, Scuffham P. Value of information analysis informing adoption and research decisions in a portfolio of health care interventions. Med Decis Making Pol Pract. 2016. https://doi.org/10.1177/2381468316642238.

6. Steuten L, van de Wetering G, Groothuis-Oudshoorn K, Retel V. A systematic and critical review of the evolving methods and applications of value of information in academia and practice. Pharmacoeconomics. 2013;31(1):25-48.

7. Tuffaha HW, Gordon LG, Scuffham PA. Value of information analysis in healthcare: a review of principles and applications. J Med Econ. 2014;17(6):377-83.

8. Tuffaha HW, El Saifi N, Chambers SK, Scuffham PA. Directing research funds to the right research projects: a review of criteria used by research organisations in Australia in prioritising health research projects for funding. BMJ Open. 2018;8:e26207. https ://doi.org/10.1136/bmjopen-2018-026207.

9. Cartier Y, Creatore MI, Hoffman SJ, Potvin L. Priority-setting in public health research funding organisations: an exploratory qualitative study among five high-profile funders. Health Res Policy Syst. 2018;16(1):53.

10. NHMRC Principles of Peer Review. https://www.nhmrc.gov. au/grants-funding/peer-review/nhmrc-principles-peer-review. Accessed 01 Dec 2017.

11. The COHRED Working Group on Priority Setting. A manual for research priority setting using the ENHR Strategy. http://www. cohred.org/downloads/578.pdf. Accessed 30 June 2018.

12. Rudan I, Gibson J, Kapiriri L, Lansang MA, Hyder AA, Lawn J, Darmstadt GL, Cousens S, Bhutta ZA, Brown KH, et al. Setting priorities in global child health research investments: assessment of principles and practice. Croatian Med J. 2007;48(5):595-604.

13. Viergever RF, Olifson S, Ghaffar A, Terry RF. A checklist for health research priority setting: nine common themes of good practice. Health Res Policy Syst. 2010;8(1):36.

14. Koffijberg H, Rothery C, Chalkidou K, Grutters J. Value of information choices that influence estimates: a systematic review of prevailing considerations. Med Decis Making. 2018;38(7):888-900.

15. Claxton K, Posnett J. An economic approach to clinical trial design and research priority-setting. Health Econ. 1996;5(6):513-24.

16. Claxton $\mathrm{K}$. The irrelevance of inference: a decision-making approach to the stochastic evaluation of health care technologies. J Health Econ. 1999;18(3):341-64.

17. Claxton KP, Sculpher MJ. Using value of information analysis to prioritise health research: some lessons from recent UK experience. Pharmacoeconomics. 2006;24(11):1055-68.

18. Grimm SE, Dixon S, Stevens JW. Assessing the expected value of research studies in reducing uncertainty and improving implementation dynamics. Med Decis Making. 2017;37(5):523-33.

19. Andronis L, Barton P. Adjusting estimates of the expected value of information for implementation: theoretical framework and practical application. Med Decis Making. 2015;36(3):296-307. 
20. Willan AR, Eckermann S. Optimal clinical trial design using value of information methods with imperfect implementation. Health Econ. 2010;19(5):549-61.

21. Hoomans T, Fenwick EAL, Palmer S, Claxton K. Value of information and value of implementation: application of an analytic framework to inform resource allocation decisions in metastatic hormone-refractory prostate cancer. Value Health. 2009; 12(2):315-24.

22. Fenwick E, Claxton K, Sculpher M. The value of implementation and the value of information: combined and uneven development. Med Decis Making. 2008;28(1):21-32.

23. Thokala P, Devlin N, Marsh K, Baltussen R, Boysen M, Kalo Z, Longrenn T, Mussen F, Peacock S, Watkins J, et al. Multiple criteria decision analysis for health care decision making - an introduction: report 1 of the ISPOR MCDA emerging good practices task force. Value Health. 2016;19(1):1-13.

24. Hansen P, Devlin N. Multi-criteria decision analysis (MCDA) in healthcare decision-making. Oxford: Oxford University Press; 2019.

25. Marsh K, MIJ, Thokala P, Baltussen R, Boysen M, Kalo Z, Lonngren T, Mussen F, Peacock S, Watkins J et al. Multiple criteria decision analysis for health care decision making - emerging good practices: report 2 of the ISPOR MCDA emerging good practices task force. Value Health 2016;19(2):125-137.

26. McKenna $\mathrm{C}$, Claxton $\mathrm{K}$. Addressing adoption and research design decisions simultaneously: the role of value of sample information analysis. Med Decis Making. 2011;31(6):853-65.

27. Claxton K. Exploring uncertainty in cost-effectiveness analysis. Pharmacoeconomics. 2008;26(9):781-98.

28. Minelli C, Baio G. Value of information: a tool to improve research prioritization and reduce waste. PLoS Med. 2015;12(9):e1001882.

29. Spiegelhalter DJ, Myles JP, Jones DR, Abrams KR. Bayesian methods in health technology assessment: a review. Health Technol Assess. 2000;4(38):1-130.

30. Ades AE, Lu G, Claxton $\mathrm{K}$. Expected value of sample information calculations in medical decision modeling. Med Decis Making. 2004;24(2):207-27.

31. Brennan A, Kharroubi S, O'Hagan A, Chilcott J. Calculating partial expected value of perfect information via Monte Carlo sampling algorithms. Med Decis Making. 2007;27(4):448-70.

32. Meltzer DO, Hoomans T, Chung JW, Basu A. Minimal modeling approaches to value of information analysis for health research. Med Decis Making. 2011;31(6):E1-22.

33. Bennette CS, Veenstra DL, Basu A, Baker LH, Ramsey SD, Carlson JJ. Development and evaluation of an approach to using value of information analyses for real-time prioritization decisions within SWOG, a large cancer clinical trials cooperative group. Med Decis Making. 2016;36(5):641-51.

34. Eckermann S, Willan AR. Time and expected value of sample information wait for no patient. Value Health. 2008;11(3):522-6.

35. Eckermann S, Karnon J, Willan A. The value of value of information best informing research design and prioritization using current methods. Pharmacoeconomics. 2010;28(9):699-709.

36. Tuffaha HW, Reynolds H, Gordon LG, Rickard CM, Scuffham PA. Value of information analysis optimizing future trial design from a pilot study on catheter securement devices. Clin Trials. 2014;11(6):648-56.

37. Chambers SK, Hyde MK, Laurie K, Legg M, Frydenberg M, Davis ID, Lowe A, Dunn J. Experiences of Australian men diagnosed with advanced prostate cancer: a qualitative study. BMJ Open. 2018;8(2):e019917.

38. Zajdlewicz L, Hyde MK, Lepore SJ, Gardiner RA, Chambers SK. Health-related quality of life after the diagnosis of locally advanced or advanced prostate cancer: a longitudinal study. Cancer Nurs. 2017;40(5):412-9.
39. Chambers SK, Girgis A, Occhipinti S, Hutchison S, Turner J, McDowell M, Mihalopoulos C, Carter R, Dunn JC. A randomized trial comparing two low-intensity psychological interventions for distressed patients with cancer and their caregivers. Oncol Nurs Forum. 2014;41(4):E256-66.

40. Chatterton ML, Chambers S, Occhipinti S, Girgis A, Dunn J, Carter R, Shih S, Mihalopoulos C. Economic evaluation of a psychological intervention for high distress cancer patients and carers: costs and quality-adjusted life-years. Psycho-Oncology. 2016;25(7):857-64.

41. Prostate cancer statistics. https://prostate-cancer.canceraustralia .gov.au/statistics. Accessed 30 June 2018.

42. Ahmed SR, Ellah MA, Mohamed OA, Eid HM. Prepregnancy obesity and pregnancy outcome. Int J Health Sci. 2009;3(2):203-8.

43. Alanis MC, Villers MS, Law TL, Steadman EM, Robinson CJ. Complications of cesarean delivery in the massively obese parturient. Am J Obstetr Gynecol 2010;203(3):271 e271-277.

44. Johnson A, Young D, Reilly J. Caesarean section surgical site infection surveillance. J Hosp Infect. 2006;64(1):30-5.

45. Opoien HK, Valbo A, Grinde-Andersen A, Walberg M. Postcesarean surgical site infections according to CDC standards: rates and risk factors. A prospective cohort study. Acta obstetricia et gynecologica Scandinavica 2007;86(9):1097-1102.

46. Masden D, Goldstein J, Endara M, Xu K, Steinberg J, Attinger C. Negative pressure wound therapy for at-risk surgical closures in patients with multiple comorbidities: a prospective randomized controlled study. Ann Surg. 2012;255(6):1043-7.

47. Chaboyer W, Anderson V, Webster J, Sneddon A, Thalib L, Gillespie BM. Negative pressure wound therapy on surgical site infections in women undergoing elective caesarean sections: a pilot RCT. Healthcare. 2014;2(4):417-28.

48. Australian Institute of Health and Welfare, Australian refined diagnosis-related groups (AR-DRG) 2011. http://www.aihw.gov. au/hospitals-data/ar-drg-data-cubes/\#versions. Accessed 30 June 2018.

49. Australian Institute of Health and Welfare, Australia's mothers and babies. http://www.aihw.gov.au/mothers-and-babies/. Acessed 30 June 2018.

50. Chalmers I, Bracken MB, Djulbegovic B, Garattini S, Grant J, Gülmezoglu AM, Howells DW, Ioannidis JPA, Oliver S. How to increase value and reduce waste when research priorities are set. The Lancet 383(9912):156-165.

51. Tuffaha HW, Scuffham PA. The Australian managed entry scheme: are we getting it right? Pharmacoeconomics. 2018;36(5):555-65.

52. Tuffaha HW, Strong M, Gordon L, Scuffham P. Efficient value of information calculation using a non-parametric regression approach: an applied perspective. Value Health. 2016;19(4):505-9.

53. Expected health benefits of additional evidence: a research prioritisation tool. http://www.york.ac.uk/che/research/teehta/prioritisa tion. Accessed 01 Feb 2015.

54. Value of Information for Cardiovascular Trials and Other Comparative Research (VICTOR). https://sop.washington.edu/choice/ research/research-projects/victor/. Accessed 01 May 2019.

55. Claxton K, Griffin S, Koffijberg H, McKenna C. How to estimate the health benefits of additional research and changing clinical practice. BMJ (Clinical research ed). 2015;351:h5987.

56. Soares MO, Sharples L, Morton A, Claxton K, Bojke L. Experiences of structured elicitation for model-based cost-effectiveness analyses. Value Health. 2018;21(6):715-23.

57. Wilson ECF, Usher-Smith JA, Emery J, Corrie PG, Walter FM. Expert elicitation of multinomial probabilities for decisionanalytic modeling: an application to rates of disease progression in undiagnosed and untreated Melanoma. Value Health. 2018;21(6):669-76 